\title{
PROLONGATION OF HOLOMORPHIC VECTOR FIELDS ON A TUBE DOMAIN
}

\author{
SATORU SHIMIZU \\ (Received May 24, 2012, revised November 27, 2012)
}

\begin{abstract}
In this paper, we discuss some algebraic criterion on the completeness of holomorphic vector fields on a tube domain $T_{\Omega}$. Our objects of consideration are polynomial vector fields on $T_{\Omega}$. We give a method of determining the higher degree complete polynomial vector fields from the data on the lower degree complete polynomial vector fields, which we call prolongation. Furthermore, we give its applications to the holomorphic equivalence problem for tube domains.
\end{abstract}

Introduction. A well-known theorem of $\mathrm{H}$. Cartan says that the group $\operatorname{Aut}(D)$ of all holomorphic automorphisms of a bounded domain $D$ in $C^{n}$ forms a Lie group with respect to the compact-open topology. This fact is fundamental in the study of complex bounded domains. Since the Lie algebra of the Lie group $\operatorname{Aut}(D)$ can be identified canonically with the Lie algebra of all complete holomorphic vector fields on $D$, the completeness of vector fields occupies an important position in the study. In general, a judgement on the completeness of vector fields is rather difficult. In fact, given a vector field, the problem of whether its integral curve $x(t)$ is defined for all $t \in \boldsymbol{R}$ has complicated aspects as the problem on the solutions of autonomous systems in the theory of nonlinear oscillations. But, in some geometric setting, there is a nice algebraic criterion on the completeness of vector fields. The purpose of this paper is to give such a criterion in the case of holomorphic vector fields on a tube domain. Our objects of consideration are polynomial vector fields on a tube domain $T_{\Omega}$. We give a method of determining higher degree complete polynomial vector fields on $T_{\Omega}$ from the data on lower degree complete polynomial vector fields on $T_{\Omega}$, which we call prolongation.

This paper is organized as follows. In Section 1, we recall basic concepts and results on tube domains, and moreover, give an explanation of the holomorphic equivalence problem for tube domains that motivates our study. Our result on prolongation of complete polynomial vector fields on a tube domain, which we call Prolongation Theorem, is given in Section 2. In Section 3, we exhibit the concrete process of prolongation through some typical examples. Finally, in Section 4, related to the holomorphic equivalence problem for tube domains, we give some remarks, and discuss applications of Prolongation Theorem. In particular, we give an answer to the holomorphic equivalence problem for tube domains admitting only affine automorphisms.

2000 Mathematics Subject Classification. Primary 32A07; Secondary 32M05.

Key words and phrases. Tube domains, Holomorphic vector fields.

Partly supported by the Grant-in-Aid for Scientific Research (C), Japan Society for the Promotion of Science. 
1. Preliminaries and background facts. We first recall some notation and terminology. An automorphism of a complex manifold $M$ means a biholomorphic mapping of $M$ onto itself. The group of all automorphisms of $M$ is denoted by Aut $(M)$. The complex manifold $M$ is called homogeneous if $\operatorname{Aut}(M)$ acts transitively on $M$. We denote by $G L(n, \boldsymbol{R}) \ltimes \boldsymbol{C}^{n}$ the subgroup of $\operatorname{Aut}\left(C^{n}\right)$ consisting of all transformations of the form

$$
C^{n} \ni z \mapsto A z+\beta \in C^{n},
$$

where $A \in G L(n, \boldsymbol{R})$ and $\beta \in \boldsymbol{C}^{n}$. Two complex manifolds are said to be holomorphically equivalent if there is a biholomorphic mapping between them. For a Lie group $G$, we denote by $G^{\circ}$ the identity component of $G$ and by Lie $G$ the Lie algebra of $G$. If $E=\{\cdot\}$ is a subset of a vector space $V$ over a field $\boldsymbol{F}$, the linear subspace of $V$ spanned by $E$ is denoted by $E_{\boldsymbol{F}}=\{\cdot\}_{\boldsymbol{F}}$. The symbol $\delta_{i j}$ denotes the Kronecker's delta.

We now recall basic concepts and results on tube domains. A tube domain $T_{\Omega}$ in $\boldsymbol{C}^{n}$ is a domain in $\boldsymbol{C}^{n}$ given by $T_{\Omega}=\boldsymbol{R}^{n}+\sqrt{-1} \Omega$, where $\Omega$ is a domain in $\boldsymbol{R}^{n}$ and is called the base of $T_{\Omega}$. Clearly, each element $\xi \in \boldsymbol{R}^{n}$ gives rise to an automorphism $\sigma_{\xi} \in \operatorname{Aut}\left(T_{\Omega}\right)$ defined by

$$
\sigma_{\xi}(z)=z+\xi \quad \text { for } z \in T_{\Omega} .
$$

Write $\Sigma=\boldsymbol{R}^{n}$. The additive group $\Sigma$ acts as a group of automorphisms on $T_{\Omega}$ by

$$
\xi \cdot z=\sigma_{\xi}(z) \text { for } \xi \in \Sigma \text { and } z \in T_{\Omega} .
$$

The subgroup of $\operatorname{Aut}\left(T_{\Omega}\right)$ induced by $\Sigma$ is denoted by $\Sigma_{T_{\Omega}}$. Note that if $\varphi \in G L(n, \boldsymbol{R}) \ltimes \boldsymbol{C}^{n}$, then $\varphi\left(T_{\Omega}\right)$ is a tube domain in $\boldsymbol{C}^{n}$, and we have $\varphi \Sigma_{T_{\Omega}} \varphi^{-1}=\Sigma_{T_{\Xi}}$, where $T_{\Xi}=\varphi\left(T_{\Omega}\right)$.

Consider a biholomorphic mapping $\varphi T_{\Omega_{1}} \rightarrow T_{\Omega_{2}}$ between two tube domains $T_{\Omega_{1}}$ and $T_{\Omega_{2}}$ in $\boldsymbol{C}^{n}$. Then, by what we have noted above and [6, Section 1, Proposition], $\varphi$ is given by an element of $G L(n, \boldsymbol{R}) \ltimes \boldsymbol{C}^{n}$ if and only if $\varphi$ is equivariant with respect to the $\Sigma$-actions. Biholomorphic mappings between tube domains equivariant with respect to the $\Sigma$-actions may be considered as natural isomorphisms in the category of tube domains. In view of this observation, we say that two tube domains $T_{\Omega_{1}}$ and $T_{\Omega_{2}}$ in $C^{n}$ are affinely equivalent if there is a biholomorphic mapping between them given by an element of $G L(n, \boldsymbol{R}) \ltimes \boldsymbol{C}^{n}$.

If the convex hull of the base $\Omega$ of a tube domain $T_{\Omega}$ in $\boldsymbol{C}^{n}$ contains no complete straight lines, then $T_{\Omega}$ is holomorphically equivalent to a bounded domain in $C^{n}$ and, by a well-known theorem of $\mathrm{H}$. Cartan, the group $\operatorname{Aut}\left(T_{\Omega}\right)$ of all automorphisms of $T_{\Omega}$ forms a Lie group with respect to the compact-open topology. The Lie algebra $\mathfrak{g}\left(T_{\Omega}\right)$ of the Lie group $\operatorname{Aut}\left(T_{\Omega}\right)$ can be identified canonically with the finite-dimensional real Lie algebra consisting of all complete holomorphic vector fields on $T_{\Omega}$.

Let $z_{j}=x_{j}+\sqrt{-1} y_{j}, j=1, \ldots, n$, be the complex coordinate functions of $\boldsymbol{C}^{n}$, where $x_{j}, y_{j} \in \boldsymbol{R}, j=1, \ldots, n$. For $z=\left(z_{1}, \ldots, z_{n}\right)$, we write $\operatorname{Re} z=\left(x_{1}, \ldots, x_{n}\right)$ and $\operatorname{Im} z=\left(y_{1}, \ldots, y_{n}\right)$. We write $\partial_{j}=\partial / \partial z_{j}$ for $j=1, \ldots, n$. Let $D$ be a domain in $\boldsymbol{C}^{n}$. Then every holomorphic vector field $Z$ on $D$ can be written in the form

$$
Z=\sum_{j=1}^{n} f_{j}(z) \partial_{j},
$$


where $f_{1}(z), \ldots, f_{n}(z)$ are holomorphic functions on $D$. The vector field $Z$ is called a polynomial vector field if $f_{1}(z), \ldots, f_{n}(z)$ are polynomials in $z_{1}, \ldots, z_{n}$. The maximum value of the degrees of the polynomials $f_{1}(z), \ldots, f_{n}(z)$ is called the degree of $Z$ and written as $\operatorname{deg} Z$. The following result is fundamental in our study.

Structure TheOrem ([6, Section 2, Theorem]). To each tube domain $T_{\Omega}$ in $\boldsymbol{C}^{n}$ whose base $\Omega$ has the convex hull containing no complete straight lines, there is associated a tube domain $T_{\tilde{\Omega}}$ which is affinely equivalent to $T_{\Omega}$ such that $\mathfrak{g}\left(T_{\tilde{\Omega}}\right)$ has the direct sum decomposition

$$
\mathfrak{g}\left(T_{\tilde{\Omega}}\right)=\mathfrak{p}+\mathfrak{e}
$$

for which

$$
\begin{aligned}
\mathfrak{p} & =\left\{X \in \mathfrak{g}\left(T_{\tilde{\Omega}}\right) ; X \text { is a polynomial vector field }\right\}, \\
\mathfrak{e} & =\sum_{i=1}^{r}\left\{e^{z_{i}}\left(\partial_{i}+\sum_{j=r+1}^{n} \sqrt{-1} a_{i}^{j} \partial_{j}\right), e^{-z_{i}}\left(\partial_{i}-\sum_{j=r+1}^{n} \sqrt{-1} a_{i}^{j} \partial_{j}\right)\right\}_{\boldsymbol{R}},
\end{aligned}
$$

where $r$ is an integer between 0 and $n$ and $a_{i}^{j}, i=1, \ldots, r, j=r+1, \ldots, n$, are real constants.

The integer $r$ is called the exponential rank of the tube domain $T_{\Omega}$, and is denoted by $e\left(T_{\Omega}\right)$. This is well-defined, because it is readily verified that if two tube domains $T_{\Omega_{1}}$ and $T_{\Omega_{2}}$ are affinely equivalent, then we have $e\left(T_{\Omega_{1}}\right)=e\left(T_{\Omega_{2}}\right)$. When a tube domain $T_{\Omega}$ satisfies $e\left(T_{\Omega}\right)=0$, we call $T_{\Omega}$ a tube domain with polynomial infinitesimal automorphisms.

Our main theme in this paper is a study of tube domains with polynomial infinitesimal automorphisms. This is motivated by the holomorphic equivalence problem for tube domains, which we will explain below.

In terms of the notion of the affine equivalence of tube domains, the holomorphic equivalence problem for them may be formulated as the problem of studying the relationship between the holomorphic and affine equivalences of tube domains. It is clear that if two tube domains in $\boldsymbol{C}^{n}$ are affinely equivalent, then they are holomorphically equivalent. What we have to ask is whether the converse assertion holds or not:

Problem. If two tube domains $T_{\Omega_{1}}$ and $T_{\Omega_{2}}$ in $C^{n}$ are holomorphically equivalent, then are they affinely equivalent?

When $\Omega_{1}$ and $\Omega_{2}$ are convex cones in $\boldsymbol{R}^{n}$, an affirmative answer is given (see Matsushima [4]). On the other hand, when $\Omega_{1}$ and $\Omega_{2}$ are arbitrary domains in $\boldsymbol{R}^{n}$ whose convex hulls contain no complete straight lines, there is a simple counterexample. In fact, consider the upper half plane

$$
T_{(0, \infty)}=\{x+\sqrt{-1} y \in \boldsymbol{C} ; x \in \boldsymbol{R}, y>0\}
$$

and the strip

$$
T_{(0, \pi)}=\{x+\sqrt{-1} y \in \boldsymbol{C} ; x \in \boldsymbol{R}, 0<y<\pi\}
$$


in the complex plane. Then the tube domains $T_{(0, \infty)}$ and $T_{(0, \pi)}$ in $C$ are holomorphically equivalent, but not affinely equivalent. We can clarify what causes a phenomenon like this by making use of the Structure Theorem stated above. It should be remarked here that Kruzhilin and Soldatkin (see [2], [3]) completely determined all tube domains in $\boldsymbol{C}^{2}$ for which holomorphic equivalence does not imply affine equivalence without imposing any assumptions on the domains.

Let $T_{\Omega_{1}}$ and $T_{\Omega_{2}}$ be tube domains in $C^{n}$ whose bases $\Omega_{1}$ and $\Omega_{2}$ have the convex hulls containing no complete straight lines. Since the exponential rank of a tube domain is an affine invariant, it is natural to reformulate the holomorphic equivalence problem for tube domains as follows:

Problem (*). If $e\left(T_{\Omega_{1}}\right)=e\left(T_{\Omega_{2}}\right)$ and if $T_{\Omega_{1}}$ and $T_{\Omega_{2}}$ are holomorphically equivalent, then are $T_{\Omega_{1}}$ and $T_{\Omega_{2}}$ affinely equivalent?

The counterexample shown above corresponds to the case where $e\left(T_{\Omega_{1}}\right) \neq e\left(T_{\Omega_{2}}\right)$, because $e\left(T_{(0, \infty)}\right)=0$ and $e\left(T_{(0, \pi)}\right)=1$. On the other hand, when $\Omega_{1}$ and $\Omega_{2}$ are bounded domains in $\boldsymbol{R}^{n}$, it is shown [8] that if $T_{\Omega_{1}}$ and $T_{\Omega_{2}}$ are holomorphically equivalent, then we have $e\left(T_{\Omega_{1}}\right)=e\left(T_{\Omega_{2}}\right)$, and $T_{\Omega_{1}}$ and $T_{\Omega_{2}}$ are affinely equivalent.

Specifying Problem $(*)$, we consider the following problem which has fundamental importance:

Problem $(* *)$. If $e\left(T_{\Omega_{1}}\right)=e\left(T_{\Omega_{2}}\right)=0$ and if $T_{\Omega_{1}}$ and $T_{\Omega_{2}}$ are holomorphically equivalent, then are $T_{\Omega_{1}}$ and $T_{\Omega_{2}}$ affinely equivalent?

When $\Omega_{1}$ and $\Omega_{2}$ are convex cones in $\boldsymbol{R}^{n}$, we have $e\left(T_{\Omega_{1}}\right)=e\left(T_{\Omega_{2}}\right)=0$ (see [4]), and an affirmative answer to Problem $(* *)$ is given, as stated above. For an attempt to solve Problem (**) in the case where $T_{\Omega_{1}}$ and $T_{\Omega_{2}}$ are arbitrary tube domains with polynomial infinitesimal automorphisms, we need a further study of the structure of $\mathfrak{g}\left(T_{\Omega}\right)$. Prolongation Theorem given in the next section enables us to make a more detailed analysis of the structure of $\mathfrak{g}\left(T_{\Omega}\right)$ and, applying this, we can give an affirmative answer to Problem (**) in various cases [7], [10], [11].

In the rest of this section, we recall some facts on the affine automorphism group of a tube domain. Let $T_{\Omega}$ be a tube domain in $\boldsymbol{C}^{n}$ whose base $\Omega$ has the convex hull containing no complete straight lines. The group $\operatorname{Aff}\left(T_{\Omega}\right)$ of all complex affine transformations of $\boldsymbol{C}^{n}$ leaving $T_{\Omega}$ invariant may be viewed as a subgroup of $\operatorname{Aut}\left(T_{\Omega}\right)$, and is called the affine automorphism group of $T_{\Omega}$. Note that $\operatorname{Aff}\left(T_{\Omega}\right)$ is a closed subgroup of the Lie group $\operatorname{Aut}\left(T_{\Omega}\right)$ and that $\Sigma_{T_{\Omega}}$ is a subgroup of $\operatorname{Aff}\left(T_{\Omega}\right)$. The subalgebra $\mathfrak{a}\left(T_{\Omega}\right)$ of $\mathfrak{g}\left(T_{\Omega}\right)$ corresponding to $\operatorname{Aff}\left(T_{\Omega}\right)$ is given by

$$
\mathfrak{a}\left(T_{\Omega}\right)=\left\{X \in \mathfrak{g}\left(T_{\Omega}\right) ; X \text { is a polynomial vector field with } \operatorname{deg} X \leq 1\right\}
$$

and the subalgebra $\mathfrak{s}\left(T_{\Omega}\right)$ of $\mathfrak{g}\left(T_{\Omega}\right)$ corresponding to $\Sigma_{T_{\Omega}}$ is given by

$$
\mathfrak{s}\left(T_{\Omega}\right)=\left\{\partial_{1}, \ldots, \partial_{n}\right\}_{\boldsymbol{R}} .
$$

Now, the group $\operatorname{Aff}(\Omega)$ of all affine transformations of $\boldsymbol{R}^{n}$ leaving $\Omega$ invariant has the structure of a Lie group in a natural manner. Let $y_{1}, \ldots, y_{n}$ be the coordinate functions of $\boldsymbol{R}^{n}$. We 
call a vector field $Y$ on $\Omega$ an affine vector field if $Y$ has the form

$$
Y=\sum_{j=1}^{n} h_{j}(y) \frac{\partial}{\partial y_{j}},
$$

where $h_{1}(y), \ldots, h_{n}(y)$ are polynomials in $y_{1}, \ldots, y_{n}$ of degree at most one. Then the Lie algebra $\mathfrak{a}(\Omega)$ of $\operatorname{Aff}(\Omega)$ can be identified canonically with the Lie algebra of all complete affine vector fields on $\Omega$. By [8, Section 1, Lemma 3], there exists a Lie algebra isomorphism $\iota_{*}$ of $\mathfrak{a}(\Omega)$ into $\mathfrak{a}\left(T_{\Omega}\right)$ such that $\mathfrak{a}\left(T_{\Omega}\right)$ is decomposed as the direct sum

$$
\mathfrak{a}\left(T_{\Omega}\right)=\mathfrak{s}\left(T_{\Omega}\right)+\iota_{*}(\mathfrak{a}(\Omega))
$$

of $\mathfrak{s}\left(T_{\Omega}\right)$ and $\iota_{*}(\mathfrak{a}(\Omega))$. In fact, $\iota_{*}: \mathfrak{a}(\Omega) \rightarrow \mathfrak{a}\left(T_{\Omega}\right)$ is given by

$$
\iota_{*}: \mathfrak{a}(\Omega) \ni \sum_{j=1}^{n}\left(\sum_{i=1}^{n} a_{j i} y_{i}+b_{j}\right) \frac{\partial}{\partial y_{j}} \mapsto \sum_{j=1}^{n}\left(\sum_{i=1}^{n} a_{j i} z_{i}+\sqrt{-1} b_{j}\right) \partial_{j} \in \mathfrak{a}\left(T_{\Omega}\right),
$$

where $a_{j i}, b_{j}, j, i=1, \ldots, n$, are real constants. As a consequence, $\mathfrak{s}\left(T_{\Omega}\right)$ is an abelian ideal in $\mathfrak{a}\left(T_{\Omega}\right)$.

2. Prolongation of complete polynomial vector fields on a tube domain. Let $T_{\Omega}$ be a tube domain in $\boldsymbol{C}^{n}$ whose base $\Omega$ is a convex domain in $\boldsymbol{R}^{n}$ containing no complete straight lines. For a polynomial vector field $Z$ on $T_{\Omega}$ of degree 2 , we write

$$
Z=\sum_{k=0}^{2}\left(X^{(k)}+\sqrt{-1} Y^{(k)}\right)
$$

where $X^{(k)}, Y^{(k)}$ are polynomial vector fields whose components with respect to $\partial_{1}, \ldots, \partial_{n}$ are homogeneous polynomials in $z_{1}, \ldots, z_{n}$ with real coefficients of degree $k$, and set

$$
Z_{[b]}=X^{(2)}+\sqrt{-1} Y^{(1)}, \quad Z_{[a]}=X^{(1)}+\sqrt{-1} Y^{(0)}, \quad Z_{[s]}=X^{(0)} .
$$

Note that $Z=Z_{[s]}+Z_{[a]}+Z_{[b]}+\sqrt{-1} Y^{(2)}$. Our criterion on the completeness of $Z$ is given in the following theorem.

Prolongation TheOREM. Let $Z$ be a polynomial vector field on $T_{\Omega}$ of degree 2 . Then $Z$ is complete on $T_{\Omega}$ if and only if one has $Y^{(2)}=0$ and the vector fields $\left[\partial_{i}, Z\right], i=$ $1, \ldots, n$, and $Z_{[a]}$ are all complete on $T_{\Omega}$. Consequently, if $Z$ is complete on $T_{\Omega}$, then $Z_{[b]}$ is complete on $T_{\Omega}$. Also, if $Z=Z_{[b]}$ and if the vector fields $\left[\partial_{i}, Z\right], i=1, \ldots, n$, are all complete on $T_{\Omega}$, then $Z$ is complete on $T_{\Omega}$.

This theorem has the following consequence, which gives a partial generalization of Murakami [5, Theorem 7.3] (cf. Remark after the corollary below).

COROLlary. Let $T_{\Omega}$ be a tube domain in $\boldsymbol{C}^{n}$ whose base $\Omega$ is a convex domain in $\boldsymbol{R}^{n}$ containing no complete straight lines. Under the assumption that every element $Z$ of $\mathfrak{g}\left(T_{\Omega}\right)$ is a polynomial vector field with $\operatorname{deg} Z \leq 2$, if $T_{\Omega}$ is homogeneous, then $T_{\Omega}$ is affinely homogeneous, that is, $\operatorname{Aff}\left(T_{\Omega}\right)$ acts transitively on $T_{\Omega}$. 
Proof OF COROLlary. We may assume without loss of generality that $T_{\Omega}$ contains the origin $o$ of $\boldsymbol{C}^{n}$, because, under a change of coordinates by a translation of $\boldsymbol{C}^{n}$, our assumption on $\mathfrak{g}\left(T_{\Omega}\right)$ is preserved. We denote by $T_{o}$ the holomorphic tangent space to $T_{\Omega}$ at $o$. Also, for a holomorphic vector field $Z$ on $T_{\Omega}$, we denote by $Z(o)$ the value of $Z$ at $o$.

We note that if $G$ is a closed subgroup of $\operatorname{Aut}\left(T_{\Omega}\right)$, then $G$ acts transitively on $T_{\Omega}$ if and only if $T_{o}=\left\{Z(o) \in T_{o} ; Z \in \mathfrak{g}\right\}$, where $\mathfrak{g}$ is the subalgebra of $\mathfrak{g}\left(T_{\Omega}\right)$ corresponding to $G$. This is a consequence of the well-known fact that if a group $H$ acts by isometries on a metric space, then every open $H$-orbit is also closed. Indeed, since $G$ acts on $T_{\Omega}$ as an isometry group with respect to the Bergman metric and since $T_{\Omega}$ is connected, it follows from this fact applied to $H=G$ that $G$ acts transitively on $T_{\Omega}$ if and only if the orbit $G \cdot o$ of $G$ through $o$ is an open submanifold of $T_{\Omega}$. Since the tangent space to $G \cdot o$ at $o$ is given by $\left\{Z(o) \in T_{o} ; Z \in \mathfrak{g}\right\}$, the latter condition can be stated as $T_{o}=\left\{Z(o) \in T_{o} ; Z \in \mathfrak{g}\right\}$.

Let $T_{o}^{\prime}$ be the subspace of $T_{o}$ consisting of all tangent vectors $Z(o)$ for which $Z$ is a complete polynomial vector field on $T_{\Omega}$ with $\operatorname{deg} Z \leq 2$. By what we noted above, under our assumption on $\mathfrak{g}\left(T_{\Omega}\right)$, if $T_{\Omega}$ is homogeneous, then we have $T_{o}=T_{o}^{\prime}$.

Now, Let $T_{o}^{\prime \prime}$ be the subspace of $T_{o}$ consisting of all tangent vectors $Z(o)$ for which $Z$ is a complete polynomial vector field on $T_{\Omega}$ with $\operatorname{deg} Z \leq 1$. Then $T_{o}^{\prime \prime}$ is the tangent space at $o$ to the orbit $\operatorname{Aff}\left(T_{\Omega}\right) \cdot o$ of the closed subgroup $\operatorname{Aff}\left(T_{\Omega}\right)$ of $\operatorname{Aut}\left(T_{\Omega}\right)$. Therefore, again by what we noted above, to prove our corollary, it is sufficient to see that $T_{o}=T_{o}^{\prime \prime}$. Let $v$ be any element of $T_{o}$. Then, by $T_{o}=T_{o}^{\prime}$, there exists a complete polynomial vector field $Z$ on $T_{\Omega}$ with $\operatorname{deg} Z \leq 2$ such that $Z(o)=v$. When $\operatorname{deg} Z \leq 1$, it is immediate that $v \in T_{o}^{\prime}$. Consider the case when $\operatorname{deg} Z=2$. In this case, as a consequence of Prolongation Theorem, we see that $Z$ has the form $Z=Z_{[s]}+Z_{[a]}+Z_{[b]}$ and $Z_{[a]}$ is complete on $T_{\Omega}$. Since $Z_{[s]} \in\left\{\partial_{1}, \ldots, \partial_{n}\right\}_{\boldsymbol{R}} \subset \mathfrak{g}\left(T_{\Omega}\right)$ and since $Z_{[s]}+Z_{[a]}$ is of degree less than or equal to 1 , it follows that $Z_{[s]}+Z_{[a]}$ is a complete polynomial vector field on $T_{\Omega}$ of degree less than or equal to 1 . On the other hand, since $Z_{[b]}(o)=0$ by the definition of $Z_{[b]}$, we have $v=Z(o)=\left(Z_{[s]}+Z_{[a]}\right)(o)$. Therefore we obtain $v \in T_{o}^{\prime \prime}$. This concludes that $T_{o}=T_{o}^{\prime \prime}$, and the proof of the corollary is completed.

REMARK. When $T_{\Omega}$ is a Siegel domain of the first kind, that is, $\Omega$ is a convex cone in $\boldsymbol{R}^{n}$, it is known (see Matsushima [4, Theorem 1]) that every element of $\mathfrak{g}\left(T_{\Omega}\right)$ is a polynomial vector field of degree less than or equal to 2 . Consequently, the above corollary applies to a Siegel domain of the first kind.

Now we begin the proof of Prolongation Theorem by recalling a general result on the completeness of vector fields. Let $D$ be a bounded domain in $\boldsymbol{C}^{n}$. Then it is known that there exists a volume element $v_{D}$ on $D$, called the Bergman volume of $D$, which is invariant under the action of $\operatorname{Aut}(D)$. We write

$$
v_{D}=(\sqrt{-1})^{n^{2}} K_{D} d z_{1} \wedge \cdots \wedge d z_{n} \wedge d \bar{z}_{1} \wedge \cdots \wedge d \bar{z}_{n}
$$


where $K_{D}$ is a positive smooth function on $D$. It is also known that

$$
d s_{D}^{2}=\sum_{i, j=1}^{n} \frac{\partial^{2} \log K_{D}}{\partial z_{i} \partial \bar{z}_{j}} d z_{i} d \bar{z}_{j}
$$

defines a Kähler metric on $D$, called the Bergman metric of $D$, which is invariant under the action of $\operatorname{Aut}(D)$. The proof of Murakami [5, Lemma 8.1] yields the following useful result on the completeness of holomorphic vector fields on $D$.

Lemma 2.1. Assume that the Kähler metric $d s_{D}^{2}$ on $D$ is complete. Then a holomorphic vector field $Z$ on $D$ is complete if and only if $L_{Z+\bar{Z}} v_{D}=0$, where $L_{Z+\bar{Z}}$ denotes the Lie derivation with respect to $Z+\bar{Z}$.

Note that, in the above lemma, if we write $Z=\sum_{j=1}^{n} f_{j} \partial_{j}$, where $f_{1}, \ldots, f_{n}$ are holomorphic functions on $D$, then $L_{Z+\bar{Z}} v_{D}$ is given by

$$
\begin{aligned}
L_{Z+\bar{Z}} v_{D}= & (\sqrt{-1})^{n^{2}} K_{D}\left\{(Z+\bar{Z}) \log K_{D}+2 \operatorname{Re}\left(\sum_{j=1}^{n} \partial_{j} f_{j}\right)\right\} \\
& \times d z_{1} \wedge \cdots \wedge d z_{n} \wedge d \bar{z}_{1} \wedge \cdots \wedge d \bar{z}_{n} .
\end{aligned}
$$

Therefore, putting

$$
Z \cdot v_{D}=(Z+\bar{Z}) \log K_{D}+2 \operatorname{Re}\left(\sum_{j=1}^{n} \partial_{j} f_{j}\right),
$$

we see that $L_{Z+\bar{Z}} v_{D}=0$ if and only if the function $Z \cdot v_{D}$ on $D$ vanishes identically on $D$. Note also that, for holomorphic vector fields $Z$ and $Z^{\prime}$ on $D$, we have

$$
\left(Z+Z^{\prime}\right) \cdot v_{D}=Z \cdot v_{D}+Z^{\prime} \cdot v_{D} .
$$

We apply Lemma 2.1 to the proof of Prolongation Theorem. For that purpose, we need some preparations. Let $T_{\Omega}$ be as in Prolongation Theorem. For $z=\left(z_{1}, \ldots, z_{n}\right) \in T_{\Omega}$, write

$$
z=x+\sqrt{-1} y, x=\left(x_{1}, \ldots, x_{n}\right) \in \boldsymbol{R}^{n}, y=\left(y_{1}, \ldots, y_{n}\right) \in \Omega,
$$

where $z_{i}=x_{i}+\sqrt{-1} y_{i}, i=1, \ldots, n$. Then the function $K_{T_{\Omega}}$ on $T_{\Omega}$ depends only on the variable $y$. Indeed, this is a consequence of the fact that the Bergman volume $v_{T_{\Omega}}$ of $T_{\Omega}$ is invariant under the action of $\operatorname{Aut}\left(T_{\Omega}\right)$, because $\operatorname{Aut}\left(T_{\Omega}\right)$ contains the group $\Sigma_{T_{\Omega}}$ of all real translations. Let $Z$ be a polynomial vector field on $T_{\Omega}$. Then we have the following basic result.

LEMMA 2.2. According to the form of $Z$, the function $Z \cdot v_{T_{\Omega}}$ is given as follows:

(i) If $Z=p z_{i} z_{j} \partial_{k}$, where $i, j, k=1, \ldots, n$, and $p \in \boldsymbol{R}$, then

$$
Z \cdot v_{T_{\Omega}}=p\left(x_{i} y_{j}+x_{j} y_{i}\right) \frac{\partial}{\partial y_{k}} \log K_{T_{\Omega}}+2 p\left(\delta_{i k} x_{j}+\delta_{j k} x_{i}\right) .
$$

(ii) If $Z=p z_{i} \partial_{j}$, where $i, j=1, \ldots, n$, and $p \in \boldsymbol{R}$, then

$$
Z \cdot v_{T_{\Omega}}=p y_{i} \frac{\partial}{\partial y_{j}} \log K_{T_{\Omega}}+2 p \delta_{i j} .
$$


(iii) If $Z=\sqrt{-1} q z_{i} \partial_{j}$, where $i, j=1, \ldots, n$, and $q \in \boldsymbol{R}$, then

$$
Z \cdot v_{T_{\Omega}}=q x_{i} \frac{\partial}{\partial y_{j}} \log K_{T_{\Omega}} .
$$

(iv) If $Z=p \partial_{i}$, where $i=1, \ldots, n$, and $p \in \boldsymbol{R}$, then

$$
Z \cdot v_{T_{\Omega}}=0 \text {. }
$$

(v) If $Z=\sqrt{-1} q \partial_{i}$, where $i=1, \ldots, n$, and $q \in \boldsymbol{R}$, then

$$
Z \cdot v_{T_{\Omega}}=q \frac{\partial}{\partial y_{i}} \log K_{T_{\Omega}} .
$$

PROOF. A straightforward computation yields the following results:

When $Z=\sum_{l=1}^{n} f_{l} \partial_{l}=p z_{i} z_{j} \partial_{k}$, where $i, j, k=1, \ldots, n$, and $p \in \boldsymbol{R}$, we have

$$
Z+\bar{Z}=p\left(x_{i} x_{j}-y_{i} y_{j}\right) \frac{\partial}{\partial x_{k}}+p\left(x_{i} y_{j}+x_{j} y_{i}\right) \frac{\partial}{\partial y_{k}}, \quad \sum_{l=1}^{n} \partial_{l} f_{l}=p\left(\delta_{i k} z_{j}+\delta_{j k} z_{i}\right) .
$$

When $Z=\sum_{l=1}^{n} f_{l} \partial_{l}=(p+\sqrt{-1} q) z_{i} \partial_{j}$, where $i, j=1, \ldots, n$, and $p, q \in \boldsymbol{R}$, we have

$$
Z+\bar{Z}=\left(p x_{i}-q y_{i}\right) \frac{\partial}{\partial x_{j}}+\left(p y_{i}+q x_{i}\right) \frac{\partial}{\partial y_{j}}, \quad \sum_{l=1}^{n} \partial_{l} f_{l}=(p+\sqrt{-1} q) \delta_{i j} .
$$

When $Z=\sum_{l=1}^{n} f_{l} \partial_{l}=(p+\sqrt{-1} q) \partial_{i}$, where $i=1, \ldots, n$, and $p, q \in \boldsymbol{R}$, we have

$$
Z+\bar{Z}=p \frac{\partial}{\partial x_{i}}+q \frac{\partial}{\partial y_{i}}, \quad \sum_{l=1}^{n} \partial_{l} f_{l}=0 .
$$

Our assertion follows from these computation results by noting that, in (2.1) applied to $D=T_{\Omega}$, the function $\log K_{T_{\Omega}}$ depends on only the variable $y$.

Let $Z$ be a polynomial vector field on $T_{\Omega}$ of degree 2 . The following lemma on the function $Z \cdot v_{T_{\Omega}}$ is proved by using Lemma 2.2, and plays a key role in our proof of Prolongation Theorem. In what follows, for a function $F$ on $T_{\Omega}$, we write

$$
F(z)=F(x, y) \text { for } z=x+\sqrt{-1} y \in T_{\Omega}, \quad x \in \boldsymbol{R}^{n}, \quad y \in \Omega .
$$

LEMmA 2.3. The functions $Z_{[b]} \cdot v_{T_{\Omega}}, Z_{[a]} \cdot v_{T_{\Omega}}, Z_{[s]} \cdot v_{T_{\Omega}}$, and $Z \cdot v_{T_{\Omega}}$ have the following properties:

(a) $\left(Z_{[b]} \cdot v_{T_{\Omega}}\right)(0, y)=0$ for all $y \in \Omega$.

(b) $\left(Z_{[a]} \cdot v_{T_{\Omega}}\right)(x, y)=u(y)$ for all $x \in \boldsymbol{R}^{n}, y \in \Omega$, where $u$ is a function on $\Omega$.

(c) $\left(Z_{[s]} \cdot v_{T_{\Omega}}\right)(x, y)=0$ for all $x \in \boldsymbol{R}^{n}, y \in \Omega$.

(d) If $Y^{(2)}=0$, i.e., $Z=Z_{[s]}+Z_{[a]}+Z_{[b]}$, then $\left(Z \cdot v_{T_{\Omega}}\right)(0, y)=u(y)$ for all $y \in \Omega$.

PROOF. Note first that $Z_{[b]}$ is given as the sum of those polynomial vector fields which has the forms stated in (i) and (iii) of Lemma 2.2, that $Z_{[a]}$ is given as the sum of those polynomial vector fields which has the forms stated in (ii) and (v) of Lemma 2.2, and that 
$Z_{[s]}$ is given as the sum of those polynomial vector fields which has the form stated in (iv) of Lemma 2.2.

We show (a). When $W$ is a polynomial vector field which has the form stated in (i) or (iii) of Lemma 2.2, it is immediate from (2.3) and (2.5) that $\left(W \cdot v_{T_{\Omega}}\right)(0, y)=0$ for all $y \in \Omega$. Therefore, our assertion is a consequence of the formula (2.2) and what we have noted first.

We show (b). When $W$ is a polynomial vector field which has the form stated in (ii) or (v) of Lemma 2.2, it is immediate from (2.4) and (2.7) that the function $\left(W \cdot v_{T_{\Omega}}\right)(x, y)$ on $T_{\Omega}$ depends only on the variable $y$. Therefore, our assertion is a consequence of the formula (2.2) and what we have noted first.

We show (c). When $W$ is a polynomial vector field which has the form stated in (iv) of Lemma 2.2, we see by (2.6) that $\left(W \cdot v_{T_{\Omega}}\right)(x, y)=0$ for all $x \in \boldsymbol{R}^{n}, y \in \Omega$. Therefore, our assertion is a consequence of the formula (2.2) and what we have noted first.

It remains to show (d). Since $Z=Z_{[s]}+Z_{[a]}+Z_{[b]}$ by assumption, it follows from (a), (b), and (c) shown above that, for all $y \in \Omega$,

$$
\left(Z \cdot v_{T_{\Omega}}\right)(0, y)=\left(Z_{[s]} \cdot v_{T_{\Omega}}\right)(0, y)+\left(Z_{[a]} \cdot v_{T_{\Omega}}\right)(0, y)+\left(Z_{[b]} \cdot v_{T_{\Omega}}\right)(0, y)=u(y) .
$$

This shows (d), and the proof of the lemma is completed.

We now prove Prolongation Theorem by applying Lemma 2.1. First of all, we note that the Bergman metric $d s_{T_{\Omega}}^{2}$ of $T_{\Omega}$ is complete on $T_{\Omega}$. Indeed, since, by assumption, the base $\Omega$ of $T_{\Omega}$ is a convex domain in $\boldsymbol{R}^{n}$ containing no complete straight lines, an argument similar to that in Kobayashi [1, (4.1.14)] shows that $T_{\Omega}$ is Carathéodory-hyperbolic and strongly complete with respect to its Carathéodory distance. Therefore the completeness of $d s_{T_{\Omega}}^{2}$ follows from [1, (4.10.19)].

To prove the "only if" part of the first assertion, suppose that $Z$ is complete on $T_{\Omega}$. It is immediate that the vector fields $\left[\partial_{i}, Z\right], i=1, \ldots, n$, are all complete on $T_{\Omega}$, since $\partial_{1}, \ldots, \partial_{n}, Z \in \mathfrak{g}\left(T_{\Omega}\right)$ and since $\mathfrak{g}\left(T_{\Omega}\right)$ forms a Lie algebra.

We show that $Y^{(2)}=0$. Write $Z=\sum_{k=0}^{2} Z^{(k)}$, where $Z^{(k)}$ is a polynomial vector field whose components with respect to $\partial_{1}, \ldots, \partial_{n}$ are homogeneous polynomials in $z_{1}, \ldots, z_{n}$ of degree $k$. Then $Z^{(2)}$ has the form

$$
Z^{(2)}=\sum_{k=1}^{n}\left(\sum_{1 \leq i \leq j \leq n} a_{i j k} z_{i} z_{j}\right) \partial_{k},
$$

where $a_{i j k}, i, j, k=1, \ldots, n, i \leq j$, are complex constants. For every $i, j=1, \ldots, n$ with $i \leq j$, we have

$$
\left[\partial_{i},\left[\partial_{j}, Z\right]\right]=\sum_{k=1}^{n}\left(1+\delta_{i j}\right) a_{i j k} \partial_{k} .
$$

On the other hand, since $\partial_{i}, \partial_{j}, Z \in \mathfrak{g}\left(T_{\Omega}\right)$ and since $\mathfrak{g}\left(T_{\Omega}\right)$ forms a Lie algebra, it follows that $\left[\partial_{i},\left[\partial_{j}, Z\right]\right]$ is in $\mathfrak{g}\left(T_{\Omega}\right)$. Therefore, we see that $a_{i j k} \in \boldsymbol{R}$ for all $k=1, \ldots, n$ in view of the fact that if $\sum_{k=1}^{n} \alpha_{k} \partial_{k} \in \mathfrak{g}\left(T_{\Omega}\right)$, where $\alpha_{1}, \ldots, \alpha_{n}$ are complex constants, then 
$\alpha_{k} \in \boldsymbol{R}$ for all $k=1, \ldots, n$ (see [6, Section 3, Lemma 5]). As a result, the coefficients $a_{i j k}, i, j, k=1, \ldots, n, i \leq j$, are all real, which implies that $Y^{(2)}=0$.

We show that $Z_{[a]}$ is complete on $T_{\Omega}$. Since $Z$ is complete, it follows from Lemma 2.1 and the remarks after it that $\left(Z \cdot v_{T_{\Omega}}\right)(x, y)=0$ for all $x \in \boldsymbol{R}^{n}, y \in \Omega$. By the fact that $Y^{(2)}=0$ shown above and the properties (d), (b) stated in Lemma 2.3 , this yields that

$$
0=\left(Z \cdot v_{T_{\Omega}}\right)(0, y)=u(y)=\left(Z_{[a]} \cdot v_{T_{\Omega}}\right)(x, y) \text { for all } x \in \boldsymbol{R}^{n}, \quad y \in \Omega .
$$

Therefore, again from Lemma 2.1 and the remarks after it, we see that $Z_{[a]}$ is complete on $T_{\Omega}$, and the "only if" part of the first assertion is proved.

To prove the "if" part of the first assertion, suppose that $Y^{(2)}=0$ and that the vector fields $\left[\partial_{i}, Z\right], i=1, \ldots, n$, and $Z_{[a]}$ are all complete on $T_{\Omega}$. It follows from Lemma 2.1

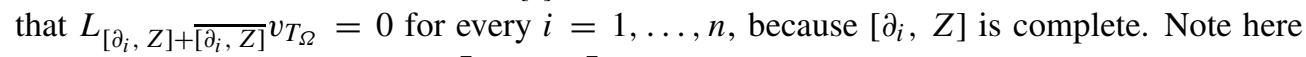
that $\left[\partial_{i}, Z\right]+\overline{\left[\partial_{i}, Z\right]}=\left[\partial_{i}+\bar{\partial}_{i}, Z+\bar{Z}\right]$, that $L_{[X, Y]}=L_{X} L_{Y}-L_{Y} L_{X}$ for vector fields $X, Y$ on $T_{\Omega}$, and that, since $v_{T_{\Omega}}$ is invariant under the action of $\Sigma_{T_{\Omega}}$, we have $L_{\partial_{i}+\bar{\partial}_{i}} v_{T_{\Omega}}=0$. Therefore, we see that

$$
\begin{aligned}
0 & =L_{\left[\partial_{i}, Z\right]+\overline{\left[\partial_{i}, Z\right]}} v_{T_{\Omega}} \\
& =L_{\left[\partial_{i}+\bar{\partial}_{i}, Z+\bar{Z}\right]} v_{T_{\Omega}} \\
& =L_{\partial_{i}+\bar{\partial}_{i}}\left(L_{Z+\bar{Z}} v_{T_{\Omega}}\right)-L_{Z+\bar{Z}}\left(L_{\partial_{i}+\bar{\partial}_{i}} v_{T_{\Omega}}\right) \\
& =L_{\partial_{i}+\bar{\partial}_{i}}\left(L_{Z+\bar{Z}} v_{T_{\Omega}}\right) \\
& =(\sqrt{-1})^{n^{2}}\left(\partial_{i}+\bar{\partial}_{i}\right)\left\{K_{T_{\Omega}}\left(Z \cdot v_{T_{\Omega}}\right)\right\} d z_{1} \wedge \cdots \wedge d z_{n} \wedge d \bar{z}_{1} \wedge \cdots \wedge d \bar{z}_{n} .
\end{aligned}
$$

In view of the fact that the function $K_{T_{\Omega}}$ depends only on the variable $y$, this implies that $\left(\partial_{i}+\bar{\partial}_{i}\right)\left(Z \cdot v_{T_{\Omega}}\right)=0$ for every $i=1, \ldots, n$, or the function $Z \cdot v_{T_{\Omega}}$ depends only on the variable $y$. As a result, we have

$$
\left(Z \cdot v_{T_{\Omega}}\right)(x, y)=\left(Z \cdot v_{T_{\Omega}}\right)(0, y) \text { for all } x \in \boldsymbol{R}^{n}, \quad y \in \Omega .
$$

On the other hand, by the assumption that $Y^{(2)}=0$ and the properties (d), (b) stated in Lemma 2.3 , we see that

$$
\left(Z \cdot v_{T_{\Omega}}\right)(0, y)=u(y)=\left(Z_{[a]} \cdot v_{T_{\Omega}}\right)(x, y) \text { for all } x \in \boldsymbol{R}^{n}, \quad y \in \Omega .
$$

Moreover, since $Z_{[a]}$ is complete on $T_{\Omega}$ by assumption, it follows from Lemma 2.1 and the remarks after it that

$$
\left(Z_{[a]} \cdot v_{T_{\Omega}}\right)(x, y)=0 \quad \text { for all } x \in \boldsymbol{R}^{n}, \quad y \in \Omega .
$$

Thus, combining (2.8), (2.9), and (2.10), we obtain

$$
0=\left(Z_{[a]} \cdot v_{T_{\Omega}}\right)(x, y)=\left(Z \cdot v_{T_{\Omega}}\right)(0, y)=\left(Z \cdot v_{T_{\Omega}}\right)(x, y) \text { for all } x \in \boldsymbol{R}^{n}, y \in \Omega .
$$

This concludes that $Z$ is complete on $T_{\Omega}$ by Lemma 2.1 and the remarks after it, and the "if" part of the first assertion is proved.

The second assertion of Prolongation Theorem is an immediate consequence of the "only if" part of the first assertion. Indeed, if $Z$ is complete, then, by the "only if" part of the 
first assertion, $Z_{[a]}$ is complete, and we have $Y^{(2)}=0$, or $Z=Z_{[s]}+Z_{[a]}+Z_{[b]}$. Since $Z_{[s]} \in\left\{\partial_{1}, \ldots, \partial_{n}\right\}_{\boldsymbol{R}} \subset \mathfrak{g}\left(T_{\Omega}\right)$, the completeness of $Z_{[b]}$ follows.

Finally, to see the third assertion of Prolongation Theorem, note that if $Z=Z_{[b]}$, then $Z_{[a]}=0$, and that the vector field $Z_{[a]}=0$ is complete on $T_{\Omega}$. Therefore, under the assumption that $Z=Z_{[b]}$, the "if" part of the first assertion implies that if the vector fields $\left[\partial_{i}, Z\right], i=1, \ldots, n$, are all complete on $T_{\Omega}$, then $Z$ is complete on $T_{\Omega}$. This completes the proof of Prolongation Theorem.

3. Examples. Prolongation Theorem shows that complete polynomial vector fields $Z$ on $T_{\Omega}$ of degree 2 are fully determined by the data on $\operatorname{Aff}\left(T_{\Omega}\right)$. Although the method of determination is purely algebraic, the completeness of vector fields is originally an analytic matter. In this section, we exhibit the concrete process of determining $Z$ through some typical examples (cf. [9, Section 2]).

EXAmPle 1. Consider the tube domain $T_{\Omega_{1}}$ in $C^{2}$ whose base $\Omega_{1}$ is given by

$$
\Omega_{1}=\left\{\left(y_{1}, y_{2}\right) \in \boldsymbol{R}^{2} ; y_{1}>y_{2}^{2}\right\} .
$$

It follows that

$$
\iota_{*}\left(\mathfrak{a}\left(\Omega_{1}\right)\right)=\left\{\left(2 \lambda z_{1}+2 \mu z_{2}\right) \partial_{1}+\left(\lambda z_{2}+\sqrt{-1} \mu\right) \partial_{2} ; \lambda, \mu \in \boldsymbol{R}\right\} .
$$

We put $g_{1}(z ; \lambda, \mu)=2 \lambda z_{1}+2 \mu z_{2}, g_{2}(z ; \lambda, \mu)=\lambda z_{2}+\sqrt{-1} \mu$. By Prolongation Theorem, to determine complete polynomial vector fields on $T_{\Omega_{1}}$ of degree 2 , it suffices to investigate the completeness for a polynomial vector field $Z$ on $T_{\Omega_{1}}$ which has the form $Z=X^{(2)}+$ $\sqrt{-1} Y^{(1)}$ with the notation of Section 2. Write $Z=f_{1}(z) \partial_{1}+f_{2}(z) \partial_{2}$, where $f_{1}(z), f_{2}(z)$ are polynomials in $z_{1}, z_{2}$. Prolongation Theorem asserts that $Z$ is complete on $T_{\Omega_{1}}$ if and only if both $\left[\partial_{1}, Z\right]=\partial_{1} f_{1}(z) \partial_{1}+\partial_{1} f_{2}(z) \partial_{2}$ and $\left[\partial_{2}, Z\right]=\partial_{2} f_{1}(z) \partial_{1}+\partial_{2} f_{2}(z) \partial_{2}$ belong to $\iota_{*}\left(\mathfrak{a}\left(\Omega_{1}\right)\right)$. Suppose that this condition holds. Then we have

$$
\begin{aligned}
& \partial_{1} f_{1}(z)=g_{1}(z ; \lambda, \mu), \\
& \partial_{1} f_{2}(z)=g_{2}(z ; \lambda, \mu), \\
& \partial_{2} f_{1}(z)=g_{1}\left(z ; \lambda^{\prime}, \mu^{\prime}\right), \\
& \partial_{2} f_{2}(z)=g_{2}\left(z ; \lambda^{\prime}, \mu^{\prime}\right),
\end{aligned}
$$

for some real constants $\lambda, \mu, \lambda^{\prime}, \mu^{\prime}$. Write $f_{2}(z)=a z_{1}^{2}+b z_{1} z_{2}+c z_{2}^{2}+\sqrt{-1}\left(p z_{1}+q z_{2}\right)$, where $a, b, c, p, q$ are real constants. Since $g_{2}(z ; \lambda, \mu), g_{2}\left(z ; \lambda^{\prime}, \mu^{\prime}\right)$ do not contain the term of a constant multiple of $z_{1}$, it follows from (3.2) and (3.4) that $a=b=0$, or

$$
f_{2}(z)=c z_{2}^{2}+\sqrt{-1}\left(p z_{1}+q z_{2}\right) .
$$

Therefore $\partial_{1} f_{2}(z), \partial_{2} f_{2}(z)$ are given by

$$
\begin{gathered}
\partial_{1} f_{2}(z)=\sqrt{-1} p, \\
\partial_{2} f_{2}(z)=2 c z_{2}+\sqrt{-1} q .
\end{gathered}
$$


The equation (3.6) implies that $\partial_{1} f_{2}(z)=g_{2}(z ; 0, p)$, and hence, by $(3.1)$, we have $\partial_{1} f_{1}(z)=$ $g_{1}(z ; 0, p)=2 p z_{2}$. We see from this equation that $f_{1}(z)$ has the form

$$
f_{1}(z)=2 p z_{1} z_{2}+c^{\prime} z_{2}^{2}+\sqrt{-1} q^{\prime} z_{2},
$$

where $c^{\prime}, q^{\prime}$ are real constants. As a consequence, $\partial_{2} f_{1}(z)$ is given by

$$
\partial_{2} f_{1}(z)=2 p z_{1}+2 c^{\prime} z_{2}+\sqrt{-1} q^{\prime} .
$$

On the other hand, the equation (3.7) implies that $\partial_{2} f_{2}(z)=g_{2}(z ; 2 c, q)$, and hence, by (3.3),

$$
\partial_{2} f_{1}(z)=g_{1}(z ; 2 c, q)=4 c z_{1}+2 q z_{2} .
$$

Hence, comparing (3.9) with (3.10), we obtain $p=2 c, c^{\prime}=q, q^{\prime}=0$. In view of (3.8) and (3.5), this shows that $f_{1}(z), f_{2}(z)$ have the form

$$
\begin{gathered}
f_{1}(z)=4 c z_{1} z_{2}+c^{\prime} z_{2}^{2}, \\
f_{2}(z)=c z_{2}^{2}+\sqrt{-1}\left(2 c z_{1}+c^{\prime} z_{2}\right) .
\end{gathered}
$$

Conversely, for any real constants $c, c^{\prime}$, if $f_{1}(z), f_{2}(z)$ are given by (3.11), (3.12), then $Z=$ $f_{1}(z) \partial_{1}+f_{2}(z) \partial_{2}$ satisfies the condition that $\left[\partial_{i}, Z\right] \in \iota_{*}\left(\mathfrak{a}\left(\Omega_{1}\right)\right), i=1,2$. In particular, putting $c=0, c^{\prime}=1$, we have $Z=z_{2}^{2} \partial_{1}+\sqrt{-1} z_{2} \partial_{2}$, which we denote by $Z_{1}$, while, putting $c=1, c^{\prime}=0$, we have $Z=4 z_{1} z_{2} \partial_{1}+\left(z_{2}^{2}+2 \sqrt{-1} z_{1}\right) \partial_{2}$, which we denote by $Z_{2}$. The real vector space of all complete polynomial vector fields $Z$ on $T_{\Omega_{1}}$ of the form $Z=X^{(2)}+\sqrt{-1} Y^{(1)}$ is spanned by $Z_{1}, Z_{2}$.

EXAMPLE 2. Consider the tube domain $T_{\Omega_{4}}$ in $C^{2}$ whose base $\Omega_{4}$ is given by

$$
\Omega_{4}=\left\{\left(y_{1}, y_{2}\right) \in \boldsymbol{R}^{2} ; y_{1}>\left|y_{2}\right|^{\rho}\right\},
$$

where $\rho$ is a real constant with $\rho>1, \rho \neq 2$. It follows that

$$
\iota_{*}\left(\mathfrak{a}\left(\Omega_{4}\right)\right)=\left\{\lambda \rho z_{1} \partial_{1}+\lambda z_{2} \partial_{2} ; \lambda \in \boldsymbol{R}\right\} .
$$

As in Example 1, let $Z$ be a polynomial vector field on $T_{\Omega_{4}}$ of the form $Z=X^{(2)}+\sqrt{-1} Y^{(1)}$, and suppose that both $\left[\partial_{1}, Z\right]$ and $\left[\partial_{2}, Z\right]$ belong to $\iota_{*}\left(\mathfrak{a}\left(\Omega_{4}\right)\right)$. Write $Z=f_{1}(z) \partial_{1}+f_{2}(z) \partial_{2}$, where $f_{1}(z), f_{2}(z)$ are polynomials in $z_{1}, z_{2}$. Then we have

$$
\begin{aligned}
& \partial_{1} f_{1}(z)=\lambda \rho z_{1}, \\
& \partial_{1} f_{2}(z)=\lambda z_{2}, \\
& \partial_{2} f_{1}(z)=\lambda^{\prime} \rho z_{1}, \\
& \partial_{2} f_{2}(z)=\lambda^{\prime} z_{2},
\end{aligned}
$$

for some real constants $\lambda, \lambda^{\prime}$. It follows from (3.13) that $f_{1}(z)$ has the form $f_{1}(z)=$ $(1 / 2) \lambda \rho z_{1}^{2}+c z_{2}^{2}+\sqrt{-1} q z_{2}$, where $c, q$ are real constants. As a consequence, $\partial_{2} f_{1}(z)$ is given by $\partial_{2} f_{1}(z)=2 c z_{2}+\sqrt{-1} q$. Comparing this with (3.15), we obtain $c=q=\lambda^{\prime}=0$, which shows

$$
f_{1}(z)=\frac{1}{2} \lambda \rho z_{1}^{2}
$$


Similarly, using (3.14) and (3.16), we see that $\lambda=0$ and

$$
f_{2}(z)=\frac{1}{2} \lambda^{\prime} z_{2}^{2}
$$

Combining (3.17), (3.18) with the fact that $\lambda=\lambda^{\prime}=0$, we conclude that $f_{1}(z)=f_{2}(z)=0$. Therefore, there exists no nonzero complete polynomial vector field $Z$ on $T_{\Omega_{4}}$ of the form $Z=X^{(2)}+\sqrt{-1} Y^{(1)}$.

EXAmple 3. Consider the tube domain $T_{\Omega_{3}^{\prime}}$ in $C^{2}$ whose base $\Omega_{3}^{\prime}$ is given by

$$
\Omega_{3}^{\prime}=\left\{\left(y_{1}, y_{2}\right) \in \boldsymbol{R}^{2} ; y_{2}>e^{y_{1}}\right\} .
$$

It follows that

$$
\iota_{*}\left(\mathfrak{a}\left(\Omega_{3}^{\prime}\right)\right)=\left\{\sqrt{-1} \lambda \partial_{1}+\lambda z_{2} \partial_{2} ; \lambda \in \boldsymbol{R}\right\} .
$$

As in Example 1, let $Z$ be a polynomial vector field on $T_{\Omega_{3}^{\prime}}$ of the form $Z=X^{(2)}+\sqrt{-1} Y^{(1)}$, and suppose that both $\left[\partial_{1}, Z\right]$ and $\left[\partial_{2}, Z\right]$ belong to $\iota_{*}\left(\mathfrak{a}\left(\Omega_{3}^{\prime}\right)\right.$ ). Write $Z=f_{1}(z) \partial_{1}+f_{2}(z) \partial_{2}$, where $f_{1}(z), f_{2}(z)$ are polynomials in $z_{1}, z_{2}$. Then we have

$$
\begin{aligned}
& \partial_{1} f_{1}(z)=\sqrt{-1} \lambda, \\
& \partial_{1} f_{2}(z)=\lambda z_{2}, \\
& \partial_{2} f_{1}(z)=\sqrt{-1} \lambda^{\prime}, \\
& \partial_{2} f_{2}(z)=\lambda^{\prime} z_{2},
\end{aligned}
$$

for some real constants $\lambda, \lambda^{\prime}$. It follows from (3.19), (3.21) that $f_{1}(z)$ has the form

$$
f_{1}(z)=\sqrt{-1}\left(p z_{1}+q z_{2}\right),
$$

where $p, q$ are real constants. As a consequence, $\partial_{1} f_{1}(z), \partial_{2} f_{1}(z)$ are given by

$$
\begin{aligned}
& \partial_{1} f_{1}(z)=\sqrt{-1} p, \\
& \partial_{2} f_{1}(z)=\sqrt{-1} q .
\end{aligned}
$$

On the other hand, it follows from (3.20), (3.22) that $f_{2}(z)$ has the form

$$
f_{2}(z)=c z_{2}^{2} \text {, }
$$

where $c$ is a real constant. As a consequence, $\partial_{1} f_{2}(z), \partial_{2} f_{2}(z)$ are given by

$$
\begin{aligned}
& \partial_{1} f_{2}(z)=0, \\
& \partial_{2} f_{2}(z)=2 c z_{2} .
\end{aligned}
$$

Comparing, respectively, (3.24), (3.25), (3.27), (3.28) with (3.19), (3.21), (3.20), (3.22), we obtain $p=\lambda=0, q=\lambda^{\prime}=2 c$. In view of (3.23) and (3.26), this shows that $f_{1}(z), f_{2}(z)$ have the form

$$
\begin{aligned}
& f_{1}(z)=2 \sqrt{-1} c z_{2}, \\
& f_{2}(z)=c z_{2}^{2} .
\end{aligned}
$$

Conversely, for any real constant $c$, if $f_{1}(z), f_{2}(z)$ are given by (3.29), (3.30), then $Z=$ $f_{1}(z) \partial_{1}+f_{2}(z) \partial_{2}$ satisfies the condition that $\left[\partial_{i}, Z\right] \in \iota_{*}\left(\mathfrak{a}\left(\Omega_{3}^{\prime}\right)\right), i=1,2$. In particular, 
putting $c=1$, we have $Z=2 \sqrt{-1} z_{2} \partial_{1}+z_{2}^{2} \partial_{2}$, which we denote by $Z_{0}$. The real vector space of all complete polynomial vector fields $Z$ on $T_{\Omega_{3}^{\prime}}$ of the form $Z=X^{(2)}+\sqrt{-1} Y^{(1)}$ is spanned by $Z_{0}$.

4. Some applications of Prolongation Theorem. In [8], to investigate the holomorphic equivalence problem for tube domains with bounded base, we needed the following criterion for a biholomorphic mapping between tube domains in $\boldsymbol{C}^{n}$ to be given by an element of $G L(n, \boldsymbol{R}) \ltimes \boldsymbol{C}^{n}$.

PROPOSITION 4.1 ([8, Section 1, Proposition 2]). Let $\varphi: T_{\Omega_{1}} \rightarrow T_{\Omega_{2}}$ be a biholomorphic mapping between two tube domains $T_{\Omega_{1}}$ and $T_{\Omega_{2}}$ in $C^{n}$ whose bases $\Omega_{1}$ and $\Omega_{2}$ have the convex hulls containing no complete straight lines, respectively. If $\varphi \operatorname{Aff}\left(T_{\Omega_{1}}\right)^{\circ} \varphi^{-1}=$ $\operatorname{Aff}\left(T_{\Omega_{2}}\right)^{\circ}$ and if $\operatorname{Aff}\left(\Omega_{i}\right)^{\circ} \subset G L(n, \boldsymbol{R}), i=1,2$, then $\varphi$ is given by an element of $G L(n, \boldsymbol{R}) \ltimes \boldsymbol{C}^{n}$.

The proof of Proposition 4.1 given in [8] yields in effect a little stronger version of the proposition.

PROPOSITION 4.2. Let $\varphi: T_{\Omega_{1}} \rightarrow T_{\Omega_{2}}$ be a biholomorphic mapping between two tube domains $T_{\Omega_{1}}$ and $T_{\Omega_{2}}$ in $C^{n}$ whose bases $\Omega_{1}$ and $\Omega_{2}$ have the convex hulls containing no complete straight lines, respectively. If the Lie algebra isomorphism $\Phi_{*}: \mathfrak{g}\left(T_{\Omega_{1}}\right) \rightarrow \mathfrak{g}\left(T_{\Omega_{2}}\right)$, given as the differential of the Lie group isomorphism

$$
\Phi: \operatorname{Aut}\left(T_{\Omega_{1}}\right) \ni \psi \mapsto \varphi \circ \psi \circ \varphi^{-1} \in \operatorname{Aut}\left(T_{\Omega_{2}}\right),
$$

maps a subalgebra $\mathfrak{t}_{1}$ of $\mathfrak{a}\left(T_{\Omega_{1}}\right)$ containing $\mathfrak{s}\left(T_{\Omega_{1}}\right)$ onto a subalgebra $\mathfrak{t}_{2}$ of $\mathfrak{a}\left(T_{\Omega_{2}}\right)$ containing $\mathfrak{s}\left(T_{\Omega_{2}}\right)$ and if $\operatorname{Aff}\left(\Omega_{i}\right)^{\circ} \subset G L(n, \boldsymbol{R}), i=1,2$, then $\varphi$ is given by an element of $G L(n, \boldsymbol{R}) \ltimes$ $C^{n}$.

This proposition enables us to give another proof for the main step in the answer by Matsushima [4, Theorem 6] to the holomorphic equivalence problem for Siegel domains of the first kind. In fact, keeping the notation of Proposition 4.2, let $T_{\Omega_{1}}$ and $T_{\Omega_{2}}$ be Siegel domains of the first kind in $\boldsymbol{C}^{n}$. The proof of [4, Theorem 6] shows that if $T_{\Omega_{1}}$ and $T_{\Omega_{2}}$ are holomorphically equivalent, then there exists a biholomorphic mapping $\varphi: T_{\Omega_{1}} \rightarrow T_{\Omega_{2}}$ between $T_{\Omega_{1}}$ and $T_{\Omega_{2}}$ such that $\Phi_{*}\left(\mathfrak{t}_{1}\right)=\mathfrak{t}_{2}$, where each $\mathfrak{t}_{i}$ is a maximal triangular subalgebra of $\mathfrak{g}\left(T_{\Omega_{i}}\right)$ satisfying the condition $\mathfrak{s}\left(T_{\Omega_{i}}\right) \subset \mathfrak{t}_{i} \subset \mathfrak{a}\left(T_{\Omega_{i}}\right)$. Since $\operatorname{Aff}\left(\Omega_{i}\right)^{\circ} \subset G L(n, \boldsymbol{R}), i=$ 1,2 as a consequence of the assumption that $T_{\Omega_{1}}$ and $T_{\Omega_{2}}$ are Siegel domains of the first kind, we can apply Proposition 4.2 to the biholomorphic mapping $\varphi: T_{\Omega_{1}} \rightarrow T_{\Omega_{2}}$, and conclude that $\varphi$ is given by an element of $G L(n, \boldsymbol{R}) \ltimes \boldsymbol{C}^{n}$. This gives another approach to the step stated in [4, pp. 266-267].

The proofs of Propositions 4.1 and 4.2 are based on the following characterization of $\mathfrak{s}\left(T_{\Omega}\right)$ as an $n$-dimensional abelian ideal in $\mathfrak{a}\left(T_{\Omega}\right)$.

LEMMA 4.1 ([8, Section 1, Lemma 4]). Let $T_{\Omega}$ be a tube domain in $C^{n}$ whose base $\Omega$ is a convex domain in $\boldsymbol{R}^{n}$ containing no complete straight lines. Suppose that $\operatorname{Aff}(\Omega)^{\circ} \subset$ 
$G L(n, \boldsymbol{R})$. If $\mathfrak{s}_{0}$ is an abelian ideal in $\mathfrak{a}\left(T_{\Omega}\right)$, then $\mathfrak{s}_{0} \subset \mathfrak{s}\left(T_{\Omega}\right)$. In particular, if moreover, $\operatorname{dim} \mathfrak{s}_{0}=n$, then $\mathfrak{s}_{0}=\mathfrak{s}\left(T_{\Omega}\right)$.

Prolongation Theorem in this paper yields another type of characterization of $\mathfrak{s}\left(T_{\Omega}\right)$.

LEMMA 4.2. Let $T_{\Omega}$ be a tube domain in $\boldsymbol{C}^{n}$ whose base $\Omega$ is a convex domain in $\boldsymbol{R}^{n}$ containing no complete straight lines. Suppose that there exists an $n$-dimensional abelian ideal $\mathfrak{s}_{0}$ in $\mathfrak{a}\left(T_{\Omega}\right)$ such that $\mathfrak{s}_{0} \neq \mathfrak{s}\left(T_{\Omega}\right)$. Then there exists a nonzero complete polynomial vector field on $T_{\Omega}$ of degree 2. In other words, under the assumption that there exists no nonzero complete polynomial vector field on $T_{\Omega}$ of degree 2 , any $n$-dimensional abelian ideal in $\mathfrak{a}\left(T_{\Omega}\right)$ must coincide with $\mathfrak{s}\left(T_{\Omega}\right)$.

PROOF. We first observe that every element $Z$ of $\mathfrak{a}\left(T_{\Omega}\right)$ can be written in the form

$$
Z=\sum_{k=1}^{n} f_{k}(z) \partial_{k}
$$

where $f_{1}(z), \ldots, f_{n}(z)$ are polynomials of degree at most one, and that

$$
\left[\partial_{i}, Z\right]=\sum_{k=1}^{n} \partial_{i} f_{k}(z) \partial_{k}, \quad i=1, \ldots, n .
$$

We now prove in steps that there exists a nonzero complete polynomial vector field on $T_{\Omega}$ of degree 2 .

We show that $\mathfrak{s}\left(T_{\Omega}\right) \cap \mathfrak{s}_{0} \neq\{0\}$. Note first that, since $\mathfrak{s}\left(T_{\Omega}\right)$ and $\mathfrak{s}_{0}$ are ideals in $\mathfrak{a}\left(T_{\Omega}\right)$, we have

$$
\left[\mathfrak{s}\left(T_{\Omega}\right), \mathfrak{s}_{0}\right] \subset \mathfrak{s}\left(T_{\Omega}\right) \cap \mathfrak{s}_{0} .
$$

Now suppose contrarily that $\mathfrak{s}\left(T_{\Omega}\right) \cap \mathfrak{s}_{0}=\{0\}$. Then we see by $(4.3)$ that $\left[\mathfrak{s}\left(T_{\Omega}\right), \mathfrak{s}_{0}\right]=\{0\}$, or

$$
\left[\partial_{i}, \mathfrak{s}_{0}\right]=\{0\}, \quad i=1, \ldots, n .
$$

Take any element $Z$ of $\mathfrak{s}_{0}$ and write $Z$ in the form (4.1). Since, by (4.4), we have $\left[\partial_{i}, Z\right]=$ $0, i=1, \ldots, n$, it follows from (4.2) that every polynomial $f_{k}(z), k=1, \ldots, n$, satisfies $\partial_{i} f_{k}(z)=0, i=1, \ldots, n$, and therefore $f_{k}(z)$ is a complex constant. By [6, Section 3, Lemma 5], we have $Z \in \mathfrak{s}\left(T_{\Omega}\right)$. This implies that $\mathfrak{s}_{0} \subset \mathfrak{s}\left(T_{\Omega}\right)$, which is a contradiction.

By what we have shown above and the assumption that $\mathfrak{s}_{0} \neq \mathfrak{s}\left(T_{\Omega}\right)$, we have $0<$ $\operatorname{dim} \mathfrak{s}\left(T_{\Omega}\right) \cap \mathfrak{s}_{0}<n$. We put $m=n-\operatorname{dim} \mathfrak{s}\left(T_{\Omega}\right) \cap \mathfrak{s}_{0}$. Applying, if necessary, a change of coordinates by a suitable element of $G L(n, \boldsymbol{R}) \ltimes \boldsymbol{C}^{n}$, we may assume that $\mathfrak{s}\left(T_{\Omega}\right) \cap \mathfrak{s}_{0}=$ $\left\{\partial_{m+1}, \ldots, \partial_{n}\right\}_{\boldsymbol{R}}$.

We show that every element $Z$ of $\mathfrak{s}_{0}$ has the form

$$
Z=\sum_{l=1}^{m} \alpha_{l} \partial_{l}+\sum_{k=m+1}^{n} f_{k}\left(z_{1}, \ldots, z_{m}\right) \partial_{k},
$$

where $\alpha_{1}, \ldots, \alpha_{m}$ are complex constants and $f_{m+1}\left(z_{1}, \ldots, z_{m}\right), \ldots, f_{n}\left(z_{1}, \ldots, z_{m}\right)$ are polynomials in $z_{1}, \ldots, z_{m}$ of degree at most one. By (4.3), we have $\left[\mathfrak{s}\left(T_{\Omega}\right), \mathfrak{s}_{0}\right] \subset\left\{\partial_{m+1}, \ldots\right.$, 
$\left.\partial_{n}\right\}_{\boldsymbol{R}}$, or equivalently

$$
\left[\partial_{i}, \mathfrak{s}_{0}\right] \subset\left\{\partial_{m+1}, \ldots, \partial_{n}\right\}_{\boldsymbol{R}}, \quad i=1, \ldots, n .
$$

Also, since $\mathfrak{s}_{0}$ is abelian, we have

$$
\left[\partial_{i}, \mathfrak{s}_{0}\right]=\{0\}, \quad i=m+1, \ldots, n .
$$

Let $Z$ be any element of $\mathfrak{s}_{0}$ and write $Z$ in the form (4.1). Since, by (4.6), we have $\left[\partial_{i}, Z\right] \in$ $\left\{\partial_{m+1}, \ldots, \partial_{n}\right\}_{\boldsymbol{R}}, i=1, \ldots, n$, it follows from (4.2) that every polynomial $f_{k}(z), k=$ $1, \ldots, m$, satisfies $\partial_{i} f_{k}(z)=0, i=1, \ldots, n$, and therefore $f_{k}(z)$ is a complex constant. Also, since, by (4.7), we have $\left[\partial_{i}, Z\right]=0, i=m+1, \ldots, n$, it follows from (4.2) that every polynomial $f_{k}(z), k=m+1, \ldots, n$, satisfies $\partial_{i} f_{k}(z)=0, i=m+1, \ldots, n$, and therefore $f_{k}(z)$ is a polynomial in $z_{1}, \ldots, z_{m}$. We thus conclude that $Z$ has the form (4.5).

In (4.5), for $l=1, \ldots, m$, write $\alpha_{l}=\alpha_{l}(Z)$. Also, for $k=m+1, \ldots, n$, denote by $\beta_{k}(Z)$ the constant term of the polynomial $f_{k}\left(z_{1}, \ldots, z_{m}\right)$. We define a real linear mapping $\tau: \mathfrak{s}_{0} \rightarrow \boldsymbol{R}^{m}$ by $\tau(Z)=\left(\operatorname{Im} \alpha_{1}(Z), \ldots, \operatorname{Im} \alpha_{m}(Z)\right)$.

We show that $\tau\left(\mathfrak{s}_{0}\right)=\boldsymbol{R}^{m}$. Let $Z$ be any element of $\mathfrak{s}_{0}$ such that $\tau(Z)=0$. Then, in (4.5), we have

$$
\alpha_{l} \in \boldsymbol{R} \text { for all } l=1, \ldots, m .
$$

Since $\sum_{l=1}^{m} \alpha_{l} \partial_{l} \in \mathfrak{g}\left(T_{\Omega}\right)$ by (4.8), it follows from (4.5) that

$$
\sum_{k=m+1}^{n} f_{k}\left(z_{1}, \ldots, z_{m}\right) \partial_{k}=Z-\sum_{l=1}^{m} \alpha_{l} \partial_{l} \in \mathfrak{g}\left(T_{\Omega}\right) .
$$

Therefore, $f_{k}\left(z_{1}, \ldots, z_{m}\right), k=m+1, \ldots, n$, are all real constants in view of the fact that if $\sum_{k=m+1}^{n} g_{k}\left(z_{1}, \ldots, z_{m}\right) \partial_{k} \in \mathfrak{g}\left(T_{\Omega}\right)$, where $g_{k}\left(z_{1}, \ldots, z_{m}\right), k=m+1, \ldots, n$, are holomorphic functions on $T_{\Omega}$, then $g_{k}\left(z_{1}, \ldots, z_{m}\right), k=m+1, \ldots, n$, are all real constants (see [6, Section 3, Lemma 6]). Combined with (4.8), this shows that the kernel ker $\tau$ of $\tau$ is given by

$$
\operatorname{ker} \tau=\mathfrak{s}_{0} \cap\left\{\partial_{1}, \ldots, \partial_{n}\right\}_{\boldsymbol{R}}=\mathfrak{s}_{0} \cap \mathfrak{s}\left(T_{\Omega}\right)=\left\{\partial_{m+1}, \ldots, \partial_{n}\right\}_{\boldsymbol{R}} .
$$

As a consequence, we have $\operatorname{dim} \operatorname{ker} \tau=n-m$. We thus obtain

$$
\operatorname{dim} \tau\left(\mathfrak{s}_{0}\right)=\operatorname{dim} \mathfrak{s}_{0}-\operatorname{dim} \operatorname{ker} \tau=n-(n-m)=m,
$$

i.e., $\tau\left(\mathfrak{s}_{0}\right)=\boldsymbol{R}^{m}$, as required.

The result of the preceding paragraph implies that, for each $l=1, \ldots, m$, there exists an element $Z_{l}^{\prime}$ of $\mathfrak{s}_{0}$ such that $\tau\left(Z_{l}^{\prime}\right)=e_{l}$, where $e_{l}$ denotes the element of $\boldsymbol{R}^{m}$ whose $l$-th component is equal to 1 and whose components except the $l$-th are all equal to 0 . We set

$$
\begin{aligned}
Z_{l}=Z_{l}^{\prime} & -\left\{\left(\operatorname{Re} \alpha_{1}\left(Z_{l}^{\prime}\right)\right) \partial_{1}+\cdots+\left(\operatorname{Re} \alpha_{m}\left(Z_{l}^{\prime}\right)\right) \partial_{m}\right\} \\
& -\left\{\left(\operatorname{Re} \beta_{m+1}\left(Z_{l}^{\prime}\right)\right) \partial_{m+1}+\cdots+\left(\operatorname{Re} \beta_{n}\left(Z_{l}^{\prime}\right)\right) \partial_{n}\right\} .
\end{aligned}
$$

Then we have $Z_{l} \in \mathfrak{g}\left(T_{\Omega}\right)$. By definition, $Z_{l}$ has the form

$$
Z_{l}=\sqrt{-1} \partial_{l}+\sum_{k=m+1}^{n} f_{k}^{(l)}\left(z_{1}, \ldots, z_{m}\right) \partial_{k},
$$


where each $f_{k}^{(l)}\left(z_{1}, \ldots, z_{m}\right), k=m+1, \ldots, n$, is a polynomial in $z_{1}, \ldots, z_{m}$ of degree at most one and the constant term of $f_{k}^{(l)}\left(z_{1}, \ldots, z_{m}\right)$ is a pure imaginary number or zero. For $l=1, \ldots, m$ and $k=m+1, \ldots, n$, write

$$
f_{k}^{(l)}\left(z_{1}, \ldots, z_{m}\right)=\sum_{i=1}^{m} a_{k ; i l} z_{i}+\sqrt{-1} \lambda_{k ; l},
$$

where $a_{k ; i l}, i, l=1, \ldots, m, k=m+1, \ldots, n$, and $\lambda_{k ; l}, l=1, \ldots, m, k=m+1, \ldots, n$, are real constants.

We show that $a_{k ; j l}=a_{k ; l j}$ for all $j, l=1, \ldots, m$ with $j \neq l$ and all $k=m+1, \ldots, n$. For $j, l=1, \ldots, m$ with $j \neq l$, consider the elements $Z_{j}$ and $Z_{l}$ of $\mathfrak{g}\left(T_{\Omega}\right)$. Noting that

$$
\begin{aligned}
& {\left[\sum_{k=m+1}^{n} f_{k}^{(j)}\left(z_{1}, \ldots, z_{m}\right) \partial_{k}, \sum_{k^{\prime}=m+1}^{n} f_{k^{\prime}}^{(l)}\left(z_{1}, \ldots, z_{m}\right) \partial_{k^{\prime}}\right]} \\
& \quad=\sum_{k, k^{\prime}=m+1}^{n}\left[f_{k}^{(j)}\left(z_{1}, \ldots, z_{m}\right) \partial_{k}, f_{k^{\prime}}^{(l)}\left(z_{1}, \ldots, z_{m}\right) \partial_{k^{\prime}}\right] \\
& \quad=\sum_{k, k^{\prime}=m+1}^{n}\left\{f_{k}^{(j)}\left(z_{1}, \ldots, z_{m}\right)\left(\partial_{k} f_{k^{\prime}}^{(l)}\left(z_{1}, \ldots, z_{m}\right)\right) \partial_{k^{\prime}}\right. \\
& \left.\quad-f_{k^{\prime}}^{(l)}\left(z_{1}, \ldots, z_{m}\right)\left(\partial_{k^{\prime}} f_{k}^{(j)}\left(z_{1}, \ldots, z_{m}\right)\right) \partial_{k}\right\} \\
& =0,
\end{aligned}
$$

we have

$$
\begin{aligned}
& {\left[Z_{j}, Z_{l}\right]} \\
& =\left[\sqrt{-1} \partial_{j}+\sum_{k=m+1}^{n} f_{k}^{(j)}\left(z_{1}, \ldots, z_{m}\right) \partial_{k}, \sqrt{-1} \partial_{l}+\sum_{k=m+1}^{n} f_{k}^{(l)}\left(z_{1}, \ldots, z_{m}\right) \partial_{k}\right] \\
& =\sqrt{-1}\left[\partial_{j}, \sum_{k=m+1}^{n} f_{k}^{(l)}\left(z_{1}, \ldots, z_{m}\right) \partial_{k}\right] \\
& -\sqrt{-1}\left[\partial_{l}, \sum_{k=m+1}^{n} f_{k}^{(j)}\left(z_{1}, \ldots, z_{m}\right) \partial_{k}\right] \\
& =\sqrt{-1} \sum_{k=m+1}^{n} a_{k ; j l} \partial_{k}-\sqrt{-1} \sum_{k=m+1}^{n} a_{k ; l j} \partial_{k} \\
& =\sqrt{-1} \sum_{k=m+1}^{n}\left(a_{k ; j l}-a_{k ; l j}\right) \partial_{k}
\end{aligned}
$$


Since $\left[Z_{j}, Z_{l}\right] \in \mathfrak{g}\left(T_{\Omega}\right)$, it follows that

$$
\sqrt{-1} \sum_{k=m+1}^{n}\left(a_{k ; j l}-a_{k ; l j}\right) \partial_{k} \in \mathfrak{g}\left(T_{\Omega}\right) .
$$

By [6, Section 3, Lemma 5], we see that $a_{k ; j l}-a_{k ; l j}=0$ for all $k=m+1, \ldots, n$, which implies our assertion.

For $k=m+1, \ldots, n$, we define a polynomial $F_{k}\left(z_{1}, \ldots, z_{m}\right)$ by

$$
F_{k}\left(z_{1}, \ldots, z_{m}\right)=\sum_{1 \leq i \leq j \leq m}\left(1-\frac{1}{2} \delta_{i j}\right) a_{k ; i j} z_{i} z_{j}+\sqrt{-1} \sum_{j=1}^{m} \lambda_{k ; j} z_{j} .
$$

The polynomials $F_{k}\left(z_{1}, \ldots, z_{m}\right), k=m+1, \ldots, n$, are of degree at most two. Note that some polynomial $F_{k}\left(z_{1}, \ldots, z_{m}\right)$ must be of degree two. Indeed, otherwise, in view of the fact that $a_{k ; i j}=a_{k ; j i}$, all the constants $a_{k ; i j}$ are equal to zero. This contradicts the assumption that $\mathfrak{s}_{0} \neq \mathfrak{s}\left(T_{\Omega}\right)$.

We show that $\partial_{l} F_{k}\left(z_{1}, \ldots, z_{m}\right)=f_{k}^{(l)}\left(z_{1}, \ldots, z_{m}\right)$ for every $l=1, \ldots, m$ and every $k=m+1, \ldots, n$. It follows that

$$
\partial_{l} F_{k}\left(z_{1}, \ldots, z_{m}\right)=\sum_{i=1}^{l-1} a_{k ; i l} z_{i}+a_{k ; l l} z_{l}+\sum_{j=l+1}^{m} a_{k ; l j} z_{j}+\sqrt{-1} \lambda_{k ; l} .
$$

Since, by what we have shown above, $a_{k ; l j}=a_{k ; j l}$ for all $j=l+1, \ldots, m$, we see that the right-hand side of this equation equals

$$
\sum_{i=1}^{l-1} a_{k ; i l} z_{i}+a_{k ; l l} z_{l}+\sum_{j=l+1}^{m} a_{k ; j l} z_{j}+\sqrt{-1} \lambda_{k ; l}=f_{k}^{(l)}\left(z_{1}, \ldots, z_{m}\right) .
$$

We thus conclude that $\partial_{l} F_{k}\left(z_{1}, \ldots, z_{m}\right)=f_{k}^{(l)}\left(z_{1}, \ldots, z_{m}\right)$.

We are now in a position to complete the proof of the lemma. We define a polynomial vector field $Z_{0}$ on $T_{\Omega}$ by

$$
Z_{0}=\sqrt{-1} \sum_{l=1}^{m} z_{l} \partial_{l}+\sum_{k=m+1}^{n} F_{k}\left(z_{1}, \ldots, z_{m}\right) \partial_{k} .
$$

To prove that there exists a nonzero complete polynomial vector field on $T_{\Omega}$ of degree 2, it is sufficient to prove that $Z_{0}$ is complete. It follows from the result of the preceding paragraph that, for every $i=1, \ldots, m$,

$$
\begin{aligned}
{\left[\partial_{i}, Z_{0}\right] } & =\sqrt{-1} \sum_{l=1}^{m} \partial_{i} z_{l} \partial_{l}+\sum_{k=m+1}^{n} \partial_{i} F_{k}\left(z_{1}, \ldots, z_{m}\right) \partial_{k} \\
& =\sqrt{-1} \partial_{i}+\sum_{k=m+1}^{n} f_{k}^{(i)}\left(z_{1}, \ldots, z_{m}\right) \partial_{k} \\
& =Z_{i}
\end{aligned}
$$


As a consequence, the vector fields $\left[\partial_{i}, Z_{0}\right], i=1, \ldots, m$, are complete on $T_{\Omega}$. On the other hand, since $\left[\partial_{i}, Z_{0}\right]=0$ for every $i=m+1, \ldots, n$, it is immediate that the vector fields $\left[\partial_{i}, Z_{0}\right], i=m+1, \ldots, n$, are complete on $T_{\Omega}$. Therefore, Prolongation Theorem shows that $Z_{0}$ is complete on $T_{\Omega}$, and the proof of the lemma is completed.

We can use Lemma 4.2 to obtain a criterion for a biholomorphic mapping between tube domains in $\boldsymbol{C}^{n}$ to be given by an element of $G L(n, \boldsymbol{R}) \ltimes \boldsymbol{C}^{n}$.

PROPOSITION 4.3. Let $\varphi: T_{\Omega_{1}} \rightarrow T_{\Omega_{2}}$ be a biholomorphic mapping between two tube domains $T_{\Omega_{1}}$ and $T_{\Omega_{2}}$ in $\boldsymbol{C}^{n}$ whose bases $\Omega_{1}$ and $\Omega_{2}$ have the convex hulls containing no complete straight lines, respectively. If $\operatorname{Aut}\left(T_{\Omega_{i}}\right)^{\circ}=\operatorname{Aff}\left(T_{\Omega_{i}}\right)^{\circ}, i=1,2$, then $\varphi$ is given by an element of $G L(n, \boldsymbol{R}) \ltimes \boldsymbol{C}^{n}$.

PROOF. We first observe that, for $i=1,2$, the group $\Sigma_{T_{\Omega_{i}}}$ is a connected Lie subgroup of $\operatorname{Aff}\left(T_{\Omega_{i}}\right)^{\circ}$, and the subalgebra $\mathfrak{s}\left(T_{\Omega_{i}}\right)$ of $\mathfrak{a}\left(T_{\Omega_{i}}\right)=\operatorname{Lie} \operatorname{Aff}\left(T_{\Omega_{i}}\right)^{\circ}$ corresponding to $\Sigma_{T_{\Omega_{i}}}$ is an $n$-dimensional abelian ideal in $\mathfrak{a}\left(T_{\Omega_{i}}\right)$.

Now, by assumption, we have

$$
\varphi \operatorname{Aff}\left(T_{\Omega_{1}}\right)^{\circ} \varphi^{-1}=\varphi \operatorname{Aut}\left(T_{\Omega_{1}}\right)^{\circ} \varphi^{-1}=\operatorname{Aut}\left(T_{\Omega_{2}}\right)^{\circ}=\operatorname{Aff}\left(T_{\Omega_{2}}\right)^{\circ} .
$$

Consequently, we can define a Lie group isomorphism $\Phi: \operatorname{Aff}\left(T_{\Omega_{1}}\right)^{\circ} \rightarrow \operatorname{Aff}\left(T_{\Omega_{2}}\right)^{\circ}$ by

$$
\Phi: \operatorname{Aff}\left(T_{\Omega_{1}}\right)^{\circ} \ni \psi \longmapsto \varphi \circ \psi \circ \varphi^{-1} \in \operatorname{Aff}\left(T_{\Omega_{2}}\right)^{\circ} .
$$

Since the differential $\Phi_{*}: \mathfrak{a}\left(T_{\Omega_{1}}\right) \rightarrow \mathfrak{a}\left(T_{\Omega_{2}}\right)$ of $\Phi$ is a Lie algebra isomorphism, we see that $\Phi_{*}\left(\mathfrak{s}\left(T_{\Omega_{1}}\right)\right)$ is an $n$-dimensional abelian ideal in $\mathfrak{a}\left(T_{\Omega_{2}}\right)$. Since the assumption $\operatorname{Aut}\left(T_{\Omega_{2}}\right)^{\circ}=$ $\operatorname{Aff}\left(T_{\Omega_{2}}\right)^{\circ}$ implies that there exists no nonzero complete polynomial vector field on $T_{\Omega_{2}}$ of degree 2, it follows from Lemma 4.2 that $\Phi_{*}\left(\mathfrak{s}\left(T_{\Omega_{1}}\right)\right)=\mathfrak{s}\left(T_{\Omega_{2}}\right)$. This shows that $\Phi\left(\Sigma_{T_{\Omega_{1}}}\right)=$ $\Sigma_{T_{\Omega_{2}}}$, or $\varphi \Sigma_{T_{\Omega_{1}}} \varphi^{-1}=\Sigma_{T_{\Omega_{2}}}$. By [6, Section 1, Proposition], we conclude that $\varphi$ is given by an element of $G L(n, \boldsymbol{R}) \ltimes \boldsymbol{C}^{n}$.

As an immediate consequence of the above proposition, we have the following corollary, which gives an answer to the holomorphic equivalence problem for tube domains admitting only affine automorphisms.

COROllary. Let $T_{\Omega_{1}}$ and $T_{\Omega_{2}}$ be two tube domains in $C^{n}$ whose bases $\Omega_{1}$ and $\Omega_{2}$ have the convex hulls containing no complete straight lines, respectively, and suppose that $\operatorname{Aut}\left(T_{\Omega_{i}}\right)^{\circ}=\operatorname{Aff}\left(T_{\Omega_{i}}\right)^{\circ}, i=1,2$. If $T_{\Omega_{1}}$ and $T_{\Omega_{2}}$ are holomorphically equivalent, then they are affinely equivalent.

\section{REFERENCES}

[1] S. KоваYAshi, Hyperbolic complex spaces, Grundlehren der Mathematischen Wissenschaften 318, SpringerVerlag, Berlin, Heidelberg, New York, 1998.

[ 2 ] N. G. KRUZhilin And P. A. Soldatkin, Affine and holomorphic equivalence of tube domains in $\boldsymbol{C}^{2}$, Math. Notes 75 (2004), 623-634.

[ 3 ] N. G. KRuZhilin And P. A. Soldatkin, Holomorphic equivalence of tube domains in $\boldsymbol{C}^{2}$, Proc. Steklov Inst. Math. 253 (2006), 90-99. 
[ 4 ] Y. Matsushima, On tube domains, in Symmetric spaces (Short Courses, Washington Univ., St. Louis, Mo., 1969-1970), pp. 255-270, Pure and Appl. Math. Vol. 8, Dekker, New York, 1972.

[ 5 ] S. MurakAmi, On automorphisms of siegel domains, Lect. Notes in Math. 286, Springer-Verlag, Berlin, Heidelberg, New York, 1972.

[6] S. ShimizU, Automorphisms of tube domains, in Geometry and Analysis on Complex Manifolds, Festschrift for Professor S. Kobayashi's 60th Birthday (T. Mabuchi, J. Noguchi, T. Ochiai, ed.), pp. 198-241, World Scientific, Singapore, New Jersey, London, Hong Kong, 1994.

[ 7 ] S. SHImizU, Holomorphic equivalence problem for two-dimensional tube domains with polynomial infinitesimal automorphisms, in Geometric Complex Analysis, Proceedings of the third international research institute, the Mathematical Society of Japan, Hayama, 1995, pp. 563-568, (J. Noguchi, H. Fujimoto, J. Kajiwara, T. Ohsawa, ed.), World Scientific, Singapore, New Jersey, London, Hong Kong, 1996.

[ 8 ] S. ShimizU, Automorphisms and equivalence of tube domains with bounded base, Math. Ann. 315 (1999), 295-320.

[ 9 ] S. ShimizU, A classification of two-dimensional tube domains, Amer. J. Math. 122 (2000), 1289-1308.

[10] S. SHIMIZU, Holomorphic equivalence problem for two-dimensional tube domains with polynomial infinitesimal automorphisms, in preparation.

[11] S. Shimizu, Structure and equivalence of a class of tube domains with solvable groups of automorphisms, in preparation.

\section{Mathematical Institute \\ TOHOKU UNIVERSITY \\ SENDAI 980-8578 \\ JAPAN}

E-mail address: shimizu@math.tohoku.ac.jp 